\title{
Conditioned tilt naming: A modified absolute judgment method is used to measure the oblique effect
}

\author{
ETHEL MATIN, ANNA DRIVAS, and VINCENT VALLE \\ C. W. Post Center, Long Island University, Greenvale, New York
}

\begin{abstract}
Two naive human observers were conditioned to report on the slant of a 3.8-cpd sinusoidal grating viewed monocularly with a light-adapted eye and presented at various orientations extending from -45 to $+135 \mathrm{deg}$. The procedures used yielded a baseline measure of performance and measures obtained during conditioning, extinction, and reconditioning of the tilt-naming response. Both subjects learned rapidly, achieving a level of performance that permits efficient measurement of meridional differences in perceived orientation (the oblique effect) at a large number of angles.
\end{abstract}

The discovery of cortical cells tuned to the orientations of lines, bars, edges, and gratings has stimulated renewed interest in psychophysical phenomena quantified with measures of perceived tilt. Included among these are the various tilt illusions and aftereffects, for which the mean of the subject's judgments (the point of subjective equality) is of primary concern. They also include the oblique effect, primarily manifested in studies of perceived tilt by poorer orientation discrimination at oblique meridians than at the horizontal or vertical. For the latter studies, the focus is on variance of the judgments-or the just noticeable difference. (See Appelle, 1972, and Wenderoth, Parkinson, \& White, 1979, for references to the literature.)

In measuring mean perceived tilt and also in measuring variance, most experimenters have required a comparison of two stimuli that are present in the visual field simultaneously. In some studies, for example, subjects report whether the two stimuli appear parallel (the parallel alignment method). In others, they adjust the position of a dot to the apparent extension of the line whose orientation is being assessed. With this line-dot method, the end of the test line and the comparison dot together produce the matching stimulus. In still other studies, an anglematching technique is employed. Each of these procedures appears to have legitimate claims as a

This research was supported by RO1 EY-02951 from the National Eye Institute and by an award from the Research Committee of C. W. Post College. We thank Laura-Lee Griffith, who assisted in processing the data and served as a subject in pilot experiments; Sherman Tatz, who commented on a preliminary version of the manuscript; and James Thoms, who assisted in photographing figures. Georgia Charalambous and Gloria Tom Wing were the cheerful and skillful subjects whose data are shown in this report. Correspondence may be addressed to Ethel Matin, Department of Psychology, C. W. Post Center, Long Island University, Greenvale, New York 11548. method for measuring perceived tilt, and it seemed a priori reasonable to expect qualitatively similar results regardless of the particular technique employed. This expectation has not been realized. Primarily, the evidence against interchangeability of method comes from the investigation of Wenderoth and his coworkers, whose data show that results depend in essential ways on the experimental technique (Emerson, Wenderoth, Curthoys, \& Edmonds, 1975; Wenderoth, Beh, \& White, 1978; Wenderoth, Parkinson, \& White, 1979).

Although the work of Wenderoth et al., perhaps by the very nature of the problem, does not permit the simple conclusion that one of these techniques is "right" and the others "wrong," their discussions appear to lean toward parallel alignment as the method of choice. But this technique shares several serious disadvantages with the other matching methods. Included among these are stimulus conditions in psychophysical experiments that are necessarily different from those used in related physiological studies, for which, of course, no matching stimuli are employed. These differences make the comparisons between physiological and psychophysical results, which modern theorizing demands, more difficult and more tenuous than they might otherwise be. Even on a purely psychophysical level, moreover, all matching techniques are inherently limited because they impose one of two undesirable strategies on the observer (or the experimenter who instructs him). On the one hand, he can make the match by moving his eyes back and forth between test stimulus and comparison stimulus, thereby producing a retinal image with unspecifiable temporal and spatial properties. Control of fixation solves that problem but produces another, in that test and comparison stimuli must then necessarily be presented to different retinal regions. A test stimulus centered on the 
fovea, for example, requires a matching stimulus placed several degrees into the periphery even when the test is small. When it is large (e.g., six cycles of a .1 -cpd grating), there is almost literally no room for a second stimulus.

The problems related to the presence of a matching stimulus are obviated with the absolute judgment methods, for which only one stimulus appears in the visual field at a given time. Nonetheless, there has been little use of the latter in the recent literature on perceived tilt. In our opinion, that neglect is justified: The absolute judgment methods are unsuitable for careful measurements because they involve uncontrolled sources of variance related to the subject's verbal behavior and past history. What is required are techniques with the advantages of the absolute judgment methods but with modifications designed to bring the subject's verbal behavior under better experimental control. This control can be achieved, we believe, with procedures that require the observer to learn the names of the orientations among which he discriminates; he does not simply use the vocabulary acquired outside the laboratory. We have chosen to call them "conditioned tilt naming" in order to focus attention on their relation to absolute judgment (sensation naming) methods and also to the conditioning methods of the animal psychophysics laboratory. More formally, the procedures we will discuss follow a paradigm that has been called a choice experiment with a one-to-one identification function (Galanter $\&$ Luce, 1963).

When the subject is at any given time required to distinguish between only two possible stimuli, conditioned sensation naming is identical to the yes-no paradigm with feedback of signal detection theory (see Green \& Swets, 1974, for a description of the theory and for extensive references to the literature). For our purposes, the "yes" and "no" categories become "counterclockwise" and "clockwise," respectively. We explored that special case in studies of the ability to discriminate within a block of trials between two stimuli differing in orientation and presented in random succession to the same retinal area (Matin \& Drivas, 1979). The judgmental task on each trial involved orientation recognition, not the pair comparison of two simultaneously present stimuli required by the matching methods. After the subject made her response, the experimenter told her the true orientation on that trial, thereby, in effect, teaching the "names" of the two stimuli used. We found that our observers were able to make reliable and sensitive discriminations, and we are persuaded by the outcome of that study as well as others currently in progress that results yielded by these procedures are entirely suitable for parametric studies of orientation discrimination. A similar conclusion about the feasibility of procedures that do not involve simultaneous matching was noted by Westheimer and his co- workers in an investigation of factors that interfere with the detection of a line's inclination from the vertical (Westheimer, Shimamura, \& McKee, 1976).

Encouraged by the finding that conditioned tilt naming for the case of two stimuli permits satisfactory measurement of orientation discrimination while obviating the difficulties inherent in the simultaneous matching procedures, we proceeded to further conditioning experiments. In particular, we were interested in studies of the multistimulus case. Such studies could provide useful data about limitations on information processing capacity (the $7 \pm 2$ phenomenon-see Garner, 1962, and Miller, 1956, for a discussion and references) and new possibilities for exploring those limitations in the orientation domain. We will outline some of the possibilities in the last section of the discussion. For the moment, we simply note that, in addition to its promise for cognitive studies of information processing capacity, the multistimulus case would also be useful in perceptual studies of illusions and aftereffects. For these, the traditional focus is on changes the inducing stimuli produce in the points of subjective equality - that is, changes in mean perceived tilt at various angles. Such changes in means cannot be measured by procedures that only involve the discriminability of two stimuli. If the subject were conditioned to a larger set of tilts, however, it would be possible to obtain preadaptation measures of the means of the response distributions at various angles and compare them with the means obtained after introduction of an inducing stimulus. Obviously, the measurements would not be made during periods when the experimenter provides feedback; by the problem's nature, he would not know what the feedback should be. However, measurements of illusory changes are logically possible during extinction following conditioning or reconditioning. ${ }^{1}$ The accuracy of such measurements would depend, of course, on the variance of the subject's reports during extinction. As will be seen in the results, that variance is markedly smaller than the baseline variance prior to conditioning of tilt names.

For the reasons outlined in the preceding paragraph, as well as others, it would be useful to have information about the statistical properties of responses made in tilt-naming experiments and to see how these responses are affected by conditioning procedures. Some relevant studies of variables affecting absolute judgments of orientation have been published (see Keene, 1963, for references). However, no data are available about either the learning that occurs with feedback or about the changes produced when feedback is subsequently withheld. The experiment described in this report was designed to obtain such data. They were gathered from two naive observers who were conditioned with a 3.8-cpd sinusoidal grating, viewed monocularly with a lightadapted eye, and presented at various orientations 
ranging from -45 to $135 \mathrm{deg}$. For both subjects, we examined the effects of conditioning (feedback presented on each trial), extinction (feedback withheld), and reconditioning of the tilt-naming response.

\section{METHOD}

\section{Subjects}

In the course of our study of conditioned tilt naming, we have worked with four students at the $\mathrm{C}$. W. Post Center of Long Island University, all of whom were naive about the literature on tilt perception and about the purpose of the experiment while it was in progress. Two of the four (L.L.G. and V.V.) were used in a preliminary study for which a luminous slit on a completely dark field was the stimulus. For the more complete set of data obtained at a later date from the other two subjects (G.C. and G.T.W.), the stimulus was a 3.8-cpd sinusoidal grating, viewed with a lightadapted eye. Only the latter data are described in this report. We wish to note, however, that satisfactory conditioning was also obtained with the very different stimulus conditions employed in the preliminary study.

\begin{abstract}
Apparatus
The monocular optical system consisted of two channels combined with a beam splitter. Light for each channel was generated by a tungsten halogen source and was diffused by opal glass. The stop in one channel was a circular hole with sharp, clean edges, visible at all times except when the sinusoidal grating in the other channel was flashed. The purpose of the blank field formed by this stop was to maintain light adaptation and to control fixation. For the second channel, the stop was a photographic transparency of a sinusoidal grating glued to a circular hole identical to the one provided in the adaptation channel. The angular position of this stop, which was mounted on a power turntable with a stepper-driven motor (Klinger Scientific, Type UR-100, P/P), could be set to an accuracy of $.05 \mathrm{deg}$ with appropriate electronic signals generated by a companion indexer unit (Klinger Scientific, Type 1T5D-CAI). This indexer was, in turn, interfaced to an Apple II microcomputer and programmed for random selection on each trial of one of the angles in the permissible set (to be described in the procedure section).

The stops in both channels were mounted $465 \mathrm{~mm}$ from the observer's eye, at which distance the field of view subtended a visual angle of $2.65 \mathrm{deg}$. By appropriate choice of neutral density filters, luminance of the adaptation field was set equal to the spaceaverage luminance of the grating $\left(34.9 \mathrm{~cd} / \mathrm{m}^{2}\right)$. The luminance calibration was performed with a United Technology meter, with the photocell placed in the position occupied by the viewing eye during the experiment. Contrast of the grating was $60 \% .^{2}$ By careful positioning of baffles to eliminate stray light and by accurate superimposition of the adaptation and test fields, we were able to eliminate any possible extraneous cues about orientation.

In addition to selecting the angular position of the grating, the computer set the intertrial time, generated warning tones that preceded presentations of stimuli, and produced pulses for gating electronic equipment that operated two shutters, one in each channel. A disk-operating system stored data on floppy disks for later processing, and a printer supplied a hard copy of each session's
\end{abstract} raw data.

\section{Procedure}

Prior to the beginning of formal experimentation, the subjects were informed of the procedures and told that the entire range of orientations was to be used, divided into 60 steps of $3 \mathrm{deg}$. Because there was no marking to identify the upper and lower ends of the stimulus, this range was $180 \mathrm{deg}$, not $360 \mathrm{deg}$. The angles chosen were $-45,-42 \ldots 129,132 \mathrm{deg}$ (Cartesian reference system, with vertical $=90 \mathrm{deg}$ ).
Each subject was run in 78 sessions, which began with an adaptation period of 2 min, during which the subject viewed the blank adaptation field. The first 12 sessions constituted a baseline, for which no feedback about the physical orientation of the stimulus was given. They were followed, in order, by 20 conditioning sessions (with trial by trial feedback), 10 sessions without feedback (Extinction 1), 12 reconditioning sessions (Reconditioning 1), 8 extinction sessions (Extinction 2), 8 reconditioning sessions (Reconditioning 2), and a final 8 extinction sessions (Extinction 3). One hundred and twenty trials were presented in each session, with an average intertrial time of $6 \mathrm{sec}$. On each trial, the experimenter typed the subject's oral response on the keyboard. Both the stimulus and response were stored in the computer's random-access memory until the end of the session, when they were transferred to a floppy disk for permanent storage. Stimulus presentations were $200 \mathrm{msec}$ in duration and were preceded by a warning sound. The subjects were asked to fixate carefully at the center of the adaptation field when the warning was delivered and to discard trials on which blinking or gross eye movements occurred when the stimulus was flashed.

Both subjects were paid hourly wages which, when conditioning sessions began, were supplemented by bonus payments calculated by the computer on the basis of the subject's performance during the session. These bonuses were printed at the end of the session and made available for the subject's examination.

\section{RESULTS}

\section{Effects of Conditioning}

Mean absolute error (mean of the absolute deviation of the subject's responses from the stimulus) is shown as a function of experimental sessions in Figures 1 and 2 for Subjects G.C. and G.T.W., respectively. These graphs show rapid learning during the first few conditioning sessions, manifested both by a decrease in mean absolute error and by a decrease in session-to-session variability for this measure of error. From the baseline data (Sessions 1-12), it seems

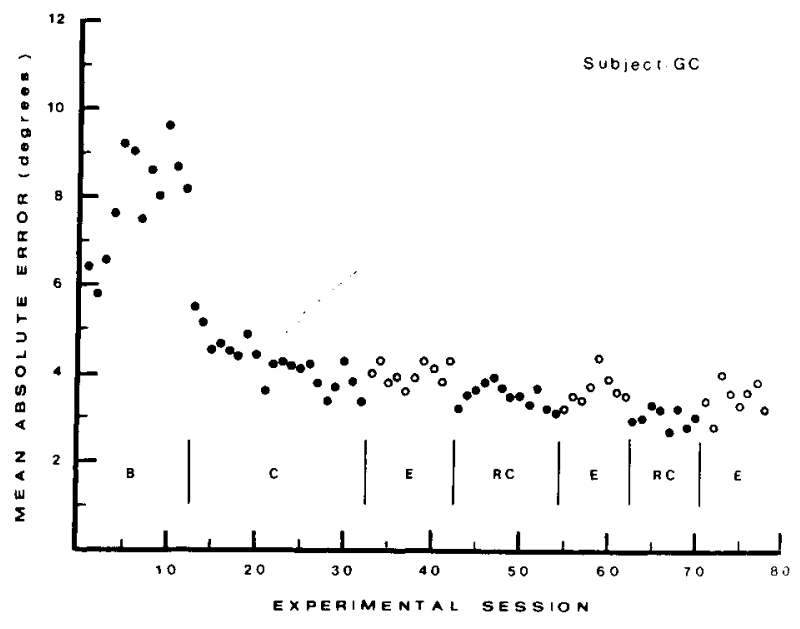

Figure 1. Mean absolute error as a function of experimental session for G.C. Twelve basellne sessions (B) were followed by 20 conditioning sessions (C), 10 sessions of extinction, 12 reconditioning sessions, 8 extinction sessions, another 8 reconditioning sessions, and a final 8 extinction sessions. Open circles represent the data points for extinction sessions, which are labeled $E$. The reconditioning sessions are labeled $\mathbf{R C}$. 


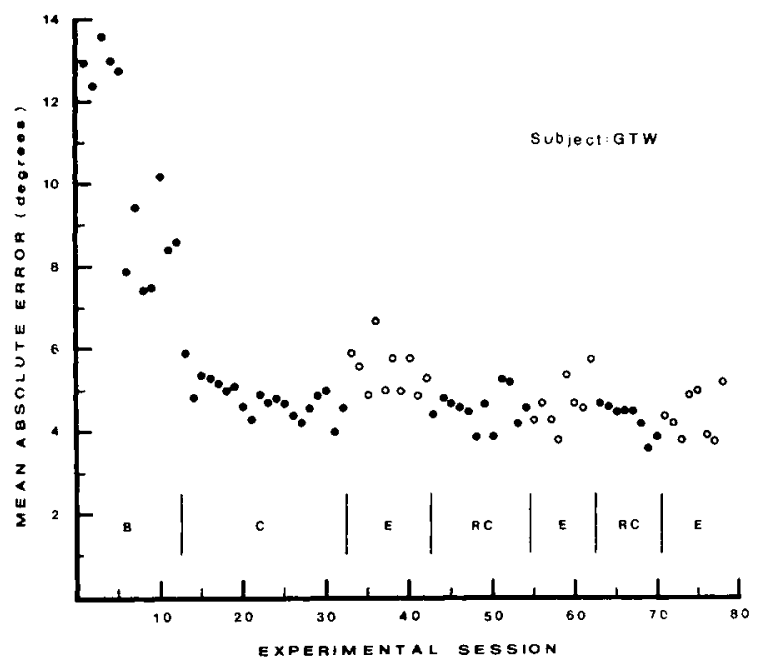

Figure 2. Mean absolute error as a function of experimental session for G.T.W. See caption to Figure 1 for details.

clear that no improvement comparable to that obtained with conditioning could be expected from simple exposure to the experimental procedures without feedback. The average absolute error of one of the two observers (G.T.W.) decreased very substantially during Session 6, but no further improvement occurred during the remaining six baseline sessions. The size of the other subject's average absolute error actually tended to increase as the number of baseline sessions increased. For both observers, moreover, a high level of session-to-session variability persisted throughout the baseline, in contrast with the dramatic decrease in this variability when feedback was introduced in the 13 th session.

Some deterioration of performance occurred during the extinction sessions. For G.T.W. this effect was most pronounced during the first extinction and tended to decrease in later extinction periods following reconditioning. The amount of deterioration was, in general, greater for this subject than for G.C. With both observers, however, errors during extinction periods were always substantially smaller than errors during the baseline.

Another measure of the effect of the conditioning procedure was obtained by calculating Shannon's average transmitted information per stimulus presentation for the baseline data and for the data from Extinction 3 (Attneave, 1959; Shannon \& Weaver, 1949). These calculations yielded baseline transmissions of 2.71 and 3.08 bits for G.T.W. and G.C., respectively. After conditioning, 3.45 bits were transmitted to G.T.W. and 3.75 to G.C. Approximately 5.9 bits $\left(\log _{2} 60\right)$ were available per stimulus presentation. (The stimulus distribution obtained from the computer's random-number generator was not perfectly uniform; hence, the amount of information was somewhat less than the 5.9 bits possible with 60 uniformly distributed stimuli.)

Each data point in Figures 1 and 2 was obtained by averaging across angles for the 120 -trial session. To show the effect of conditioning without losing this angular information, we used the mean of the signed deviations of the responses from the stimuli, and the standard deviations of the response distributions for each of the 60 angles used, averaged separately for the baseline and for the sessions in Extinction 3. These statistics are shown in Figures 3 and 5 for G.C. and in Figures 4 and 6 for G.T.W. For both subjects, pronounced reductions can be seen in the mean errors and in the standard deviations when the results in Extinction 3 are compared with those obtained during the baseline.

\section{Oblique Effect}

Large meridional differences in performance (the "oblique effect") can be seen for both the baseline data and for the results from Extinction 3 (Figures 3, 4,5 , and 6). These differences are manifested in both the mean deviation (the "constant error" of classical psychophysics) and in the standard deviation of the response distribution (the "variable error"). The latter is one possible measure of discriminability. In addition, we derived another measure by calculating slopes of the psychometric functions at various angles in the range studied. The procedure will be described in the next paragraph. For reasons to be considered in the discussion, the data for that analysis

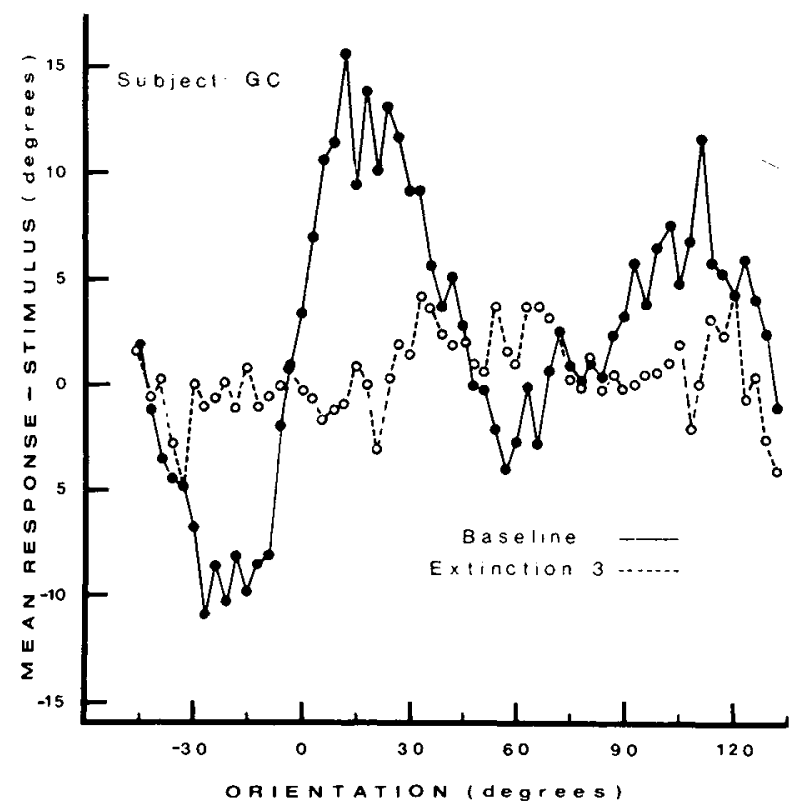

Figure 3. Comparison of G.C.'s signed error (mean response - stimulus) as a function of orientation for the baseline and for data from the last extinction period. 
were taken from the reconditioning sessions, that is, from all sessions in which feedback was given, except for the 20 conditioning sessions, during which rapid learning occurred. The entire analysis was performed by the computer with a program that read the data from the floppy disk, performed the necessary calculations, and printed a hard copy of the results.

The first step in calculating psychometric functions was to prepare a confusion matrix, a two-dimensional matrix with 3,600 cells ( 60 stimuli $\times 60$ responses), each of which contained the frequency of the stimulusresponse pair corresponding to that cell. Appropriately chosen subsets of the large matrix were used in obtaining psychometric functions centered at various angles. Assume, for example, a center angle of $90 \mathrm{deg}$. Working with the data collected at this angle and at angles \pm 3 and $\pm 6 \mathrm{deg}$ on either side of it (84, $87,90,93$, and $96 \mathrm{deg}$ ), we calculated the proportion of responses that were greater than $90 \mathrm{deg}$ (counting half of those equal to $90 \mathrm{deg}$ as greater than $90 \mathrm{deg}$, the other half as less). This procedure yielded a psychometric function (proportion of ">90 deg" responses vs. angle). After converting these proportions to standard normal scores, we fitted a linear function to the $\mathrm{z}$ score vs. stimulus distri-

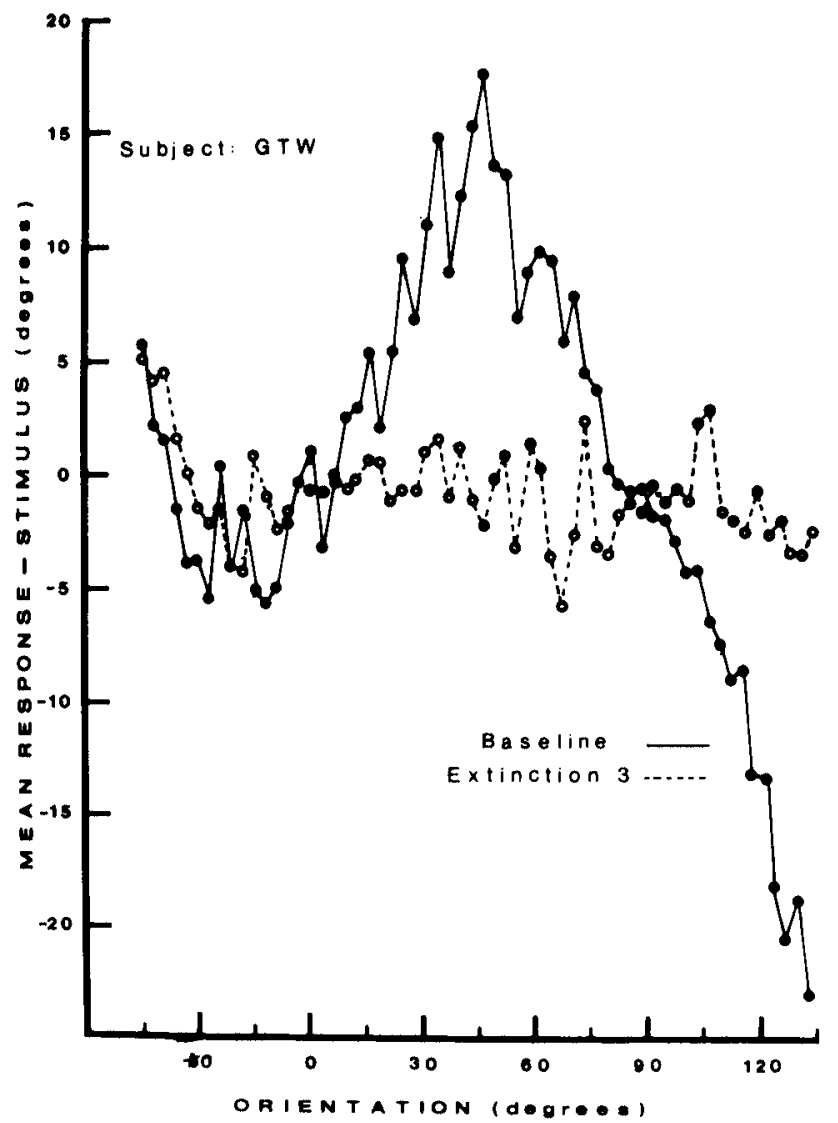

Figure 4. Signed error as a function of orientation for G.T.W. See caption to Figure 3 for details.

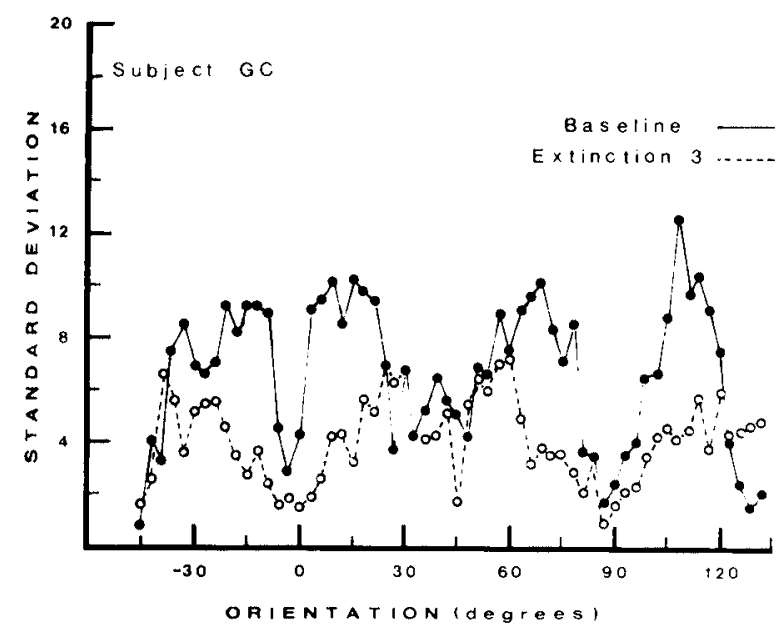

Figure 5. Comparison of standard deviations of G.C.'s response distributions as a function of orientation for the baseline and for data from the last extinction session.

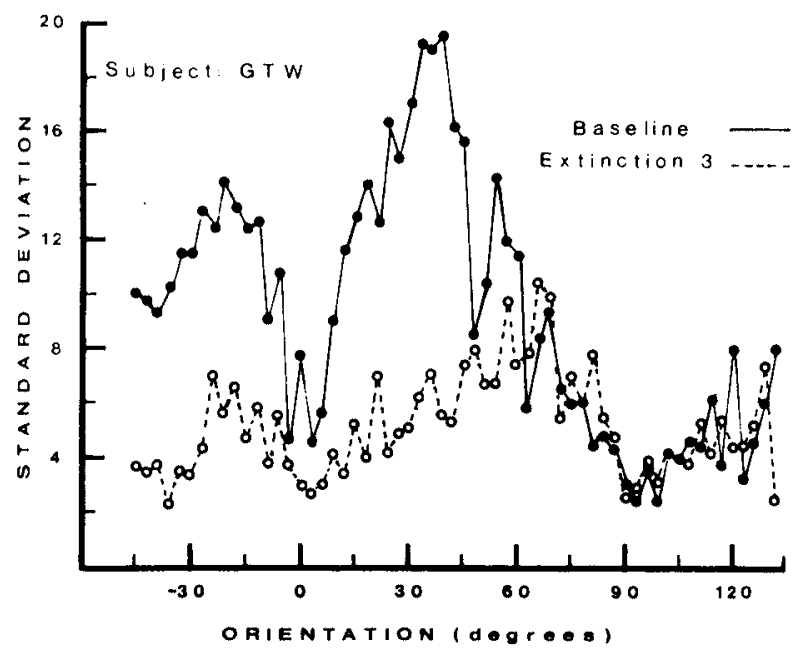

Figure 6. Standard deviation as a function of orlentation for G.T.W. See caption to Figure 5 for detalls.

bution by the method of least squares. The slope of this function was used to measure discriminability in the neighborhood of $90 \mathrm{deg}$. The results for similar calculations performed for center angles ranging from -36 to $126 \mathrm{deg}$ in steps of $3 \mathrm{deg}$ are plotted as a function of center angle in Figures 7 and 8 for Subjects G.C. and G.T.W., respectively. These plots will be considered in the discussion. For the moment, we simply remind the reader that the slope measure is directly proportional to discriminability, unlike the more traditional just noticeable difference, for which the relation is inverse.

\section{DISCUSSION}

On the continuum of techniques available to the psychophysicist, conditioned sensation naming lies 


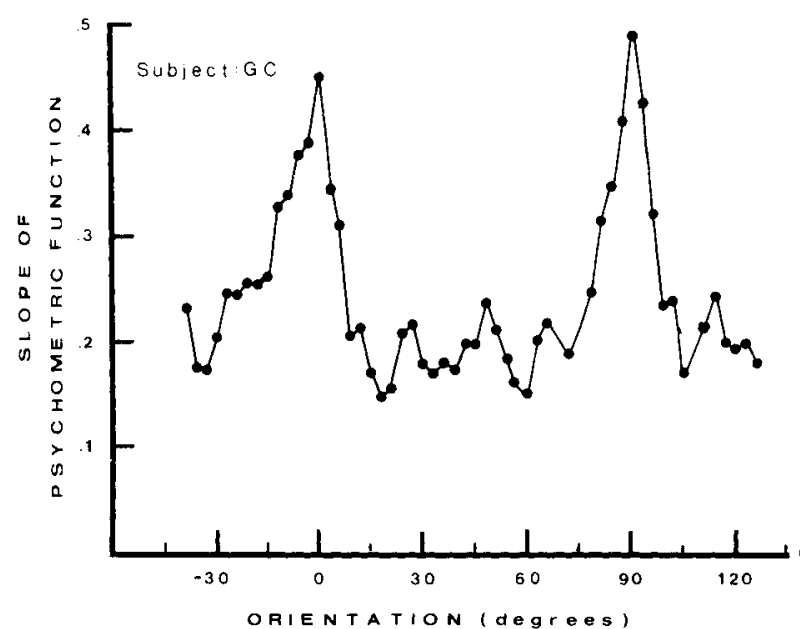

Figure 7. The oblique effect for G.C. Sensitivity to orientation, expressed as the slope of the psychometric function, is plotted as a function of orientation.

somewhere between the absolute judgment methods, for which there is very heavy reliance on the language shared by experimenter and subject, and the conditioned response methods of the animal psychophysics laboratory, for which there is no shared language at all. The tilt names we used, for example, were obviously not entirely unlearned before experimentation began. Indeed, our choice of names was intended in part to take advantage of the subject's prior familiarity with them in order to minimize learning time. Nonetheless, conditioning produced very pronounced changes in our subjects' responding. These learning effects will be considered in the first section of the discussion. In the second, we focus attention on measurements of the oblique effect.

To avoid confusion, the reader should note from the outset that we will make a distinction between discriminability and sensitivity. The former is an experimentally obtained measure, affected by sensitivity but also by other factors. That is, discriminability reflects several sources of variance, including "noise" in the stimulus (see Green \& Swets, 1974), sensitivity and sensory memory (Durlach \& Braida, 1969; Kinchla \& Smyzer, 1967), bias variance (Atkinson, 1963; Heinemann et al., 1969; Heinemann \& Chase, 1975; McCarthy \& Davison, 1981; Wickelgren, 1968), shifts of attention (Heinemann et al., 1969; Heinemann $\&$ Chase, 1975) and perhaps other factors not explicitly recognized in the psychophysical literature. It can be derived from experimental results by calculating measures like $d^{\prime}$, slope of the psychometric function, the standard deviation, the JND, etc. Within this framework, the $d^{\prime}$ measure of discriminability enjoys the important advantage of being free of mean bias, but not of bias variance.

\section{Effects of Conditioning}

In the present experiments, feedback produced changes in discriminability and also in mean response. One measure of the former can be seen in Figures 5 and 6, which show standard deviations of the response distributions as a function of stimulus orientation. Comparison of the data from the baseline with those of Extinction 3 in these figures reveals marked improvement in discriminability with conditioning. We think this improvement can be most reasonably attributed to a reduction in bias variance, that is, to a steadier criterion, not to increased sensitivity. (Another measure of discriminability, slope of the psychometric function, will be considered in the following section of the discussion.)

The effect of feedback on mean response is shown in the learning curves (Figures 1 and 2) and in the comparisons of baseline and Extinction 3 in Figures 3 and 4. As was, of course, expected, conditioning produced a shift of the mean response toward the physical orientation, that is, a shift toward the equal bias criterion defined by the feedback. To prevent misunderstanding in its interpretation, we note explicitly that the subjects were required to identify stimuli by certain experimenter-assigned names, regardless of any prior conceptions of what those names should be. Thus, for example, they were not asked to assign the name " 90 " to the stimulus that subjectively appears vertical, but rather to say " 90 " when the stimulus the experimenter called " 90 " appeared. From the consistency of their responses, we are able to make inferences about the ability to discriminate in the neighborhood of $90 \mathrm{deg}$; from a shift in mean response with inspection of an inducing stimulus, we would be able to measure the magnitude of an illusion or aftereffect. (As noted in the introduction, the latter measurements would have to be made during

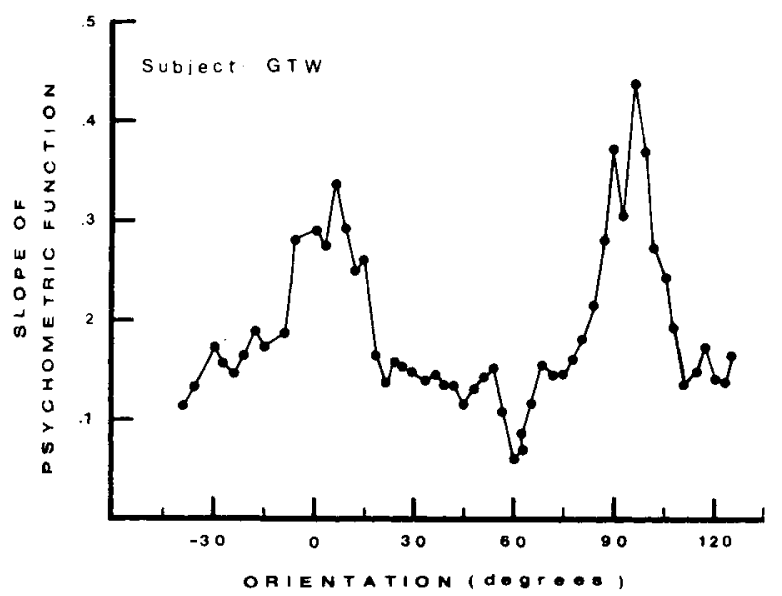

Figure 8. The oblique effect for G.T.W. See caption to Figure 7 for details. 
extinction, not during a time when the subject receives feedback.) The experiment can provide no information, however, about the physical value of the stimulus that appears vertical; the physically vertical stimulus is not necessarily (indeed, not usually) the subjectively vertical one.

\section{The Oblique Effect}

Both the conditioned and unconditioned tilt-naming data reveal meridional differences in perceived orientation. Of themselves, these differences are certainly not surprising; peaks in performance near the horizontal and vertical (the oblique effect) are found routinely with all methods of measuring perceived tilt, and peaks near $45 \mathrm{deg}$ (such as those in Figures 3 and 5) are sometimes reported with absolute judgment methods (e.g., Keene, 1963, Figure 1). New insights into this familiar phenomenon are suggested, however, by examining the changes in meridional differences that occur with conditioning.

Consider, first, the mean error (Figures 3 and 4) and note that the meridional differences are much less pronounced in Extinction 3 than in the baseline. Indeed, it would be difficult to argue on the basis of the amount of data available in this study that any systematic differences remain after the subject is conditioned. This result has no bearing, of course, on the possibility that some sensory biases toward or away from the main meridians actually exist; their presence would tend to be obscured by the conditioning procedures. Nonetheless, we are inclined to believe that the bulk of the mean error oblique effect obtained with unconditioned absolute judgments is a reflection of meridional differences in vocabulary, not in sensory capacity. Such a "vocabulary" explanation could account, for example, for the relatively good performance sometimes found at $45 \mathrm{deg}$ with the absolute judgment method (but not with the matching methods) if we assume that subjects who are not specifically conditioned to a large set of tilt names are inclined to say " 45 " for any angle in that general region. By so doing, they would create a spuriously low response error at $45 \mathrm{deg}$.

As noted earlier in the discussion, discriminability of the stimuli improved with conditioning, an effect we attributed primarily to a reduction in bias variance. Nonetheless, a pronounced oblique effect remained in the discriminability measures obtained throughout the experiment. This persisting effect can be seen for Extinction 3 in the standard deviations shown in Figures 5 and 6, which provide a useful estimate of the "noise level" that would be present if the subject's responses, after a suitable period of conditioning, were used to measure the tilt aftereffect by computing the difference between the mean response before and after inspection of an inducing stimulus. However, the extinction data should not be used when the study's purpose is simply to obtain the best possible estimate of the ability to discriminate. For such estimates, feedback is never logically contraindicated. If the ability does not exist, feedback cannot create it. Neither can feedback produce a spuriously high estimate of discriminability. In fact, the only possible effect it can have is to prevent (or to minimize) an underestimation resulting from variance due to nonsensory factors (such as poorly controlled criteria). ${ }^{3}$ The best available measures of discriminability in the present experiment are therefore those that can be derived from the data of the reconditioning sessions: At the time they were gathered, most of the learning had occurred and the continuing feedback contributed to optimal performance. Accordingly, we focus our attention on the results of those sessions, displayed as slopes of the psychometric functions in Figures 7 and 8.

We have chosen the slope measure for our further discussion of discriminability because slope (or its reciprocal, the JND) is less sensitive than the standard deviation of the response distribution at a single orientation to language-based artifacts. The standard deviation (which is the conventional measure used in previous work on absolute judgments of orientation) can assume an artifactually low value at an angle with a "popular" name if the subject simply uses that name for any angle in the region. Slopes of the psychometric functions, which are derived from responses in a neighborhood of angles, are much less susceptible to this type of error with both conditioned and unconditioned tilt naming.

Figures 7 and 8 show a very pronounced oblique effect for discriminability under our experimental conditions. Indeed, the magnitude of the effect (measured as the ratio of the steepest and most shallow slopes) is at least as great, and probably greater, than the values that have been reported with conventional matching procedures or with the signal detection procedures we have used in analyzing data when two stimuli are employed. For the latter, the mean of the slope $90 \mathrm{deg} / \mathrm{slope} 45 \mathrm{deg}$ ratio averaged across subjects in several studies (all of which were performed with the same 3.8-cpd grating used in the present experiment) was 1.75 , with a standard error of .115 . In the present study, these ratios were 2.54 and 3.4 for Subjects G.C. and G.T.W., respectively. The former subject also participated in one of the experiments using two stimuli and signal detection methods. For that study, her mean slope ratio (averaged across four experimental conditions) was 2.34 . These results suggest that the cause of the oblique effect combines interactively, not additively, with the cause of the overall poorer discriminability found with larger stimulus-response sets.

Because it supplies a convenient way of measuring discriminability at a large number of angles with a relatively small amount of data (again, see the slopes in Figures 7 and 8), conditioned tilt naming provides 
a useful tool for studies of the oblique effect. Such studies, moreover, would simultaneously provide data that could contribute to clarification of various current theories about the limitations on information processing capacity in the multistimulus case (e.g., the theories of Chase, 1982; Durlach \& Braida, 1969; Gravetter \& Lockhead, 1973; Heinemann, 1982; and Luce, Green, \& Weber, 1976). The tilt naming method could therefore be useful in the development of a cognitive psychology of perceived tilt, expanding the scope of orientation discrimination studies beyond their traditional niche in the sensation and perception categories.

\section{REFERENCE NOTE}

1. Chase, S. Multiple category classification by pigeons. Paper presented at the meetings of the Psychonomic Society, Washington, D.C., November 1977.

\section{REFERENCES}

Appelle, S. Perception and discrimination as a function of stimulus orientation: The "oblique effect" in man and animals. Psychological Bulletin, 1972, 78, 266-278.

Atkinson, R. A variable sensitivity theory of signal detection. Psychological Review, 1963, 70, 91-106.

AtTneave, F. Applications of information theory to psychology. New York: Holt, Rinehart \& Winston, 1959.

Bush, R., Galanter, E., \& Luce, R. D. Characterization and classification of choice experiments. In R. D. Luce, R. Bush, \& E. Galanter (Eds.), Handbook of mathematical psychology. New York: Wiley, 1963.

Chase, S. Pigeons and the magical number seven. In M. Commons, R. Herrnstein, \& A. Wagner (Eds.), Quantitative analyses of behavior (Vol. 4): Discrimination procedures. Cambridge, Mass: Ballinger, 1982.

Durlach, N., \& Braida, L. Intensity perception I. Preliminary theory of intensity resolution. Journal of the Acoustical Society of America, 1969, 46, 372-382.

Emerson, P., Wenderoth, P., Curthoys, I., \& Edmonds, I. Measuring perceived orientation. Vision Research, 1975, 15, 1031-1033.

GARNER, W. Uncertainty and structure as psychological concepts. New York: Wiley, 1962.

Gravetter, F., \& Lockhead, G. Criterial range as a frame of reference for stimulus judgment. Psychological Review, 1973, 80, 203-216.

Green, D. M., \& Swets, J. Signal detection theory and psychophysics. Huntington, N.Y: Krieger, 1974.

HeinemanN, E. A memory model for the acquisition of discriminations. In $\mathbf{M}$. Commons, R. Herrnstein, \& A. Wagner (Eds.), Quantitative analyses of behavior (Vol. 4): Discrimination processes. Cambridge, Mass: Ballinger, 1982.
Heinemann, E.; Avin, E., Sullivan, M., \& Chase, S. Analysis of stimulus generalization with a psychophysical method. Journal of Experimental Psychology, 1969, 80, 215-224.

Heinemann, E., \& Chase, S. Stimulus generalization. In W. Estes (Ed.), Handbook of learning and cognitive processes (Vol. 2): Conditioning and behavior theory. Hillsdale, N.J: Erlbaum, 1975.

KeEne, G. The effect of response codes on the accuracy of making absolute judgments of lineal inclinations. Journal of General Psychology, 1963, 69, 37-50.

Kinchla, R., \& Smyzer, F. A diffusion model of perceptual memory. Perception \& Psychophysics, 1967, 2, 219-229.

Luce, R. D., Green, D. M., \& Weber, D. Attention bands in absolute identification. Perception \& Psychophysics, 1976, 20 , 49-54.

Matin, E., \& Drivas, A. Acuity for orientation measured with a sequential recognition task and signal detection methods. Perception \& Psychophysics, 1979, 25, 161-168.

McCARThY, D., \& Davison, M. Towards a behavioral theory of bias in signal detection. Perception \& Psychophysics, 1981, 29, 371-382.

Miller, G. The magical number seven, plus or minus two. Psychological Review, 1956, 63, 81-97.

Shannon, C., \& WEAVER, W. The mathematical theory of communication. Urbana: University of Illinois Press, 1949.

Wendero'th, P., BEH, H., \& White, D. Perceptual distortion of an oblique line in the presence of an abutting vertical line. Vision Research, 1978, 18, 923-930.

Wenderoth, P., Parkinson, A., \& White, D. A comparison of visual tilt illusions measured by the techniques of vertical setting, parallel matching, and dot alignment. Perception, 1979, 8, 47-57.

Westheimer, G., Shimamura, K., \& McKee, S. Interference with line orientation sensitivity. Journal of the Optical Society of America, 1976, 66, 332-338.

WickelgreN, W. Unidimensional strength theory and component analysis of noise in absolute and comparative judgments. Journal of Mathematical Psychology, 1968, 5, 102-122.

\section{NOTES}

1. This suggested method for measuring an aftereffect has not been used in either the human or animal psychophysical literature. In our opinion, it has interesting potential for both. Its use in work with animals would depend, of course, on success in teaching them to discriminate among a set of several stimuli. Contrary to what is probably popular opinion, such multistimulus discriminations are feasible with appropriate conditioning procedures (Chase, 1982, and Chase, Note 1).

2. The contrast calibration was furnished by Arthur Lange and Charles F. Stromeyer, from whom we purchased the grating. We are grateful for their courtesy.

3. We assume, of course, that the experimenter takes great care to eliminate any possible extraneous cues to orientation.

(Manuscript received March 19, 1981; revision accepted for publication December 22, 1981.) 